Biomedical Physics

April 2015

\title{
Understanding the Nonlinear Dynamics of Microbubbles in Clinical Ultrasound Applications
}

Over the past decade, the use of "microbubbles" has enhanced the use of ultrasound for non-invasive diagnostic imaging. Microbubbles have also been shown to hold immense promise in the context of the site-specific delivery of drugs; for example, directly to cancer cells as well as to specific regions of the brain.

In both cases, scientists know that the "resonant oscillations" of microbubbles is an important factor determining their efficacy. Biomedical Physics PhD student Amin Jafari Sojahrood is, however, a member of the team - which includes his supervisors Dr. Raffi Karshafian and Dr. Michael Kolios - that is the first to have conducted a comprehensive study of the link between applied pressure amplitude and microbubbles' behaviour. The results of this study were recently published in Nonlinear Dynamics (Amin is the paper's lead author).

\section{Microbubbles at work}

Formally known as "ultrasound contrast agents," microbubbles are filled with gas and encased in a lipid, polymer or albumin shell. Each one measures between 1 and 8 microns (the diameter of a red blood cell is about 5 microns; that of a human hair, about 70 microns). As Amin explains, "microbubbles' acoustic response dictates the outcome of both imaging and therapeutic applications of ultrasound." With the former, "stronger backscattered signals are desirable in order to increase the detectability of vascular tissues. In drug-delivery applications, the most important thing is the shear stress microbubble oscillations exert on nearby cells; that's what we need to maximize."

Stabilizing microbubble oscillations and minimizing their destruction are important goals for both imaging and therapy. Taking the latter, for instance, stable microbubbles can generate longer-lasting shear stress on nearby cells, thereby improving the efficacy of drug delivery.

\section{Nonlinear physics}

Even though it's clear that that diagnostic and therapeutic outcomes depend on microbubble behaviour, their dynamics are not well understood. That is because of the nonlinear relationship between microbubble behaviour and three fundamental ultrasound exposure parameters - frequency, pressure and microbubble size.

Combining numerical and experimental approaches, Amin and his co-investigators used nonlinear physics methods including bifurcation diagrams (Figure 1) - to shed light on microbubbles' behaviour with a view to optimizing ultrasounds' imaging and therapeutic applications. "Ours is the first study comprehensively to take into account the effect of a decrease in microbubbles' resonance frequency as applied acoustic pressure increases," Amin says. "As a result, we were able to classify the dynamics of the resonant microbubbles."

\section{Key findings}

Using microbubbles' pressure-dependent resonance frequency, Amin and his team were able to boost substantially 9-fold - the microbubbles' detectability. "This increase," Amin explains, "occurred at lower pressures, compared to the 
conventional method of insonifying microbubbles with their linear resonance frequencies." In addition, Amin and his co-investigators were able to reduce the destruction of microbubbles by limiting the radial expansion ratio. Taken together, these outcomes offer "significant advantages for optimizing microbubble-mediated imaging and therapeutic ultrasound applications."

Funding: Vanier CIHR scholarship, NSERC Discovery Grant 